\title{
Control of Helical Navigation by Three-Dimensional Flagellar Beating
}

\author{
Dario Cortese® and Kirsty Y. Wan ${ }^{*}$ \\ Living Systems Institute and College of Engineering, Mathematics and Physical Sciences, University of Exeter, \\ Exeter EX4 4QD, United Kingdom
}

(Received 27 September 2020; revised 10 December 2020; accepted 13 January 2021; published 24 February 2021)

\begin{abstract}
Helical swimming is a ubiquitous strategy for motile cells to generate self-gradients for environmental sensing. The model biflagellate Chlamydomonas reinhardtii rotates at a constant $1-2 \mathrm{~Hz}$ as it swims, but the mechanism is unclear. Here, we show unequivocally that the rolling motion derives from a persistent, nonplanar flagellar beat pattern. This is revealed by high-speed imaging and micromanipulation of live cells. We construct a fully 3D model to relate flagellar beating directly to the free-swimming trajectories. For realistic geometries, the model reproduces both the sense and magnitude of the axial rotation of live cells. We show that helical swimming requires further symmetry breaking between the two flagella. These functional differences underlie all tactic responses, particularly phototaxis. We propose a control strategy by which cells steer toward or away from light by modulating the sign of biflagellar dominance.
\end{abstract}

DOI: 10.1103/PhysRevLett.126.088003

Organisms perceive their world as three dimensional. Biological swimmers adopt chiral or helical trajectories to navigate through bulk fluid. Helical navigation is a ubiquitous locomotion strategy found across diverse taxa and multiple length scales, from the planktonic larvae of marine invertebrates [1] to small protists [2] and spermatozoa [3]. Bacteria bias the rotation of chiral flagella in response to chemical gradients [4]. In contrast, many unicellular eukaryotes steer toward or away from external stimuli (light, chemicals, gravity) via helical klinotaxis and a corkscrewing motion around their body axis. Such organisms routinely integrate sensory information obtained by subcellular sensors (receptors, eyespots), which periodically scan the environment, with motor actuators (cilia, flagella), to adjust their swimming trajectories according to the stimulus. These self-actions can enhance signal perception at the microscale [5], with important consequences for evolution and eukaryogenesis [6]. Helical movement could help compensate for asymmetries in body shape [7] and/or filament actuation [8], but it is unclear whether such asymmetries evolved as an adaptation to helical swimming or to facilitate it in the first place. Self-propelled cholesteric liquid crystal droplets also exhibit curling and helical motions, depending on an interplay between surface flows and nematic order [9]. The complex motility strategies of real cells can facilitate the development of next-generation artificial swimmers and controllable devices [10].

Published by the American Physical Society under the terms of the Creative Commons Attribution 4.0 International license. Further distribution of this work must maintain attribution to the author(s) and the published article's title, journal citation, and DOI.
For algal flagellates, the control of flagellar beating is key to effective spatial navigation and reorientation to photostimuli [11-13]. Spermatozoa steer via changes to their 3D beat pattern [14], while ciliates tilt the orientation of ciliary fields to turn [15]. Chlamydomonas swims with two near-identical flagella using a characteristic breaststroke, rotating slowly $(1-2 \mathrm{~Hz})$ about its axis as it swims along left-handed helices [16,17]. Each $(\sim 10 \mu \mathrm{m})$ cell has a unique photosensor (eyespot), fixed in position on the rigid cell body [18]. The eyespot scans the environment and modulates phototactic turning by periodic shading $[19,20]$. Yet to date, there has been no compelling explanation for this characteristic helical motion. Here, we combine theory and experiment to reveal how Chlamydomonas, despite a very symmetric body plan, manipulates nonplanar biflagellar beating to achieve helical swimming and stimulus-dependent steering responses in 3D. In contrast, other microswimmers rely on obvious body asymmetries to steer [21], such as two distinct flagella types in some dinoflagellates [22].

Model formulation.-Our fully 3D model of the swimmer comprises three beads interacting hydrodynamically in an incompressible Newtonian fluid at zero Reynolds number. The cell body is a sphere of radius $a_{0}$, located at $\boldsymbol{r}_{0}$. Two smaller beads (radius $a \ll a_{0}$ ) are localized at the centers of drag of the flagella $\boldsymbol{r}_{i}, i=1$, 2. Flagella beads are driven by variable tangential forces $F_{i}^{(t)}, i=1,2$ and constrained along circular orbits of radius $R$ by normal components $F_{i}^{(n)}$, mimicking breaststroke swimming. All three beads are constrained on a rigid triangular scaffold at rest with the body frame of reference (Fig. 1), with dimensions $\ell$ and $h$, and $a \ll a_{0} \ll h, \ell$. This is motivated by experiments that show that flow fields induced by freely swimming Chlamydomonas cells are 


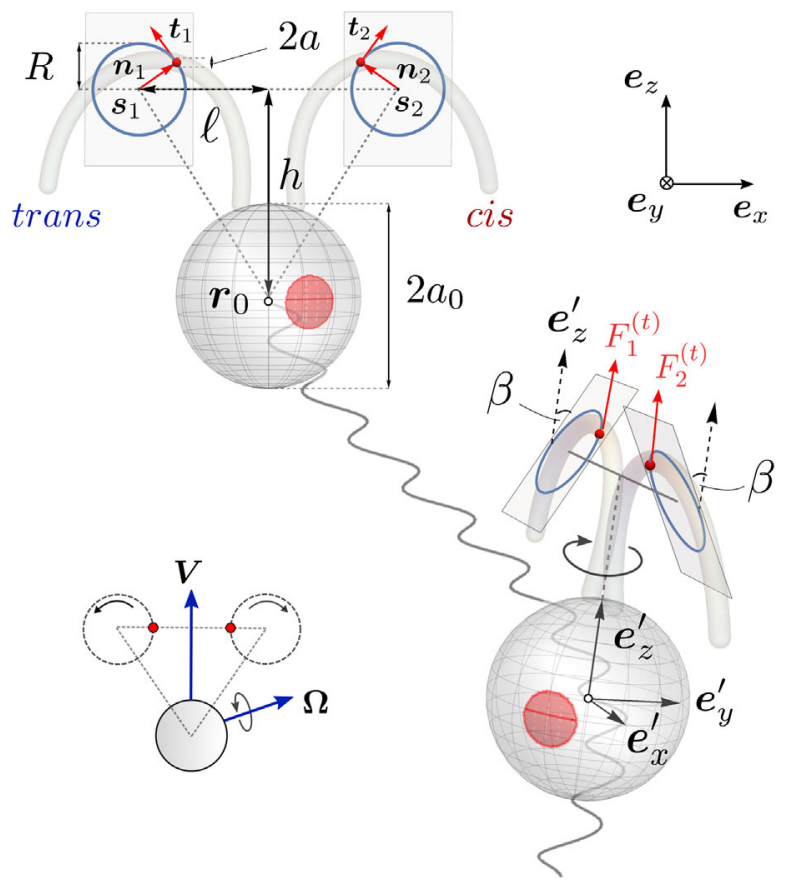

FIG. 1. A fully 3D model of a freely swimming Chlamydomonas cell, in front and side views. Flagellar beating is modeled by small beads constrained to rotate along circular orbits embedded within a pair of tilted beat planes, for a realistic scaffold geometry (inset). $\left\{\boldsymbol{e}_{x}^{\prime}, \boldsymbol{e}_{y}^{\prime}, \boldsymbol{e}_{z}^{\prime}\right\}$ and $\left\{\boldsymbol{e}_{x}, \boldsymbol{e}_{y}, \boldsymbol{e}_{z}\right\}$ are the body and laboratory reference frames.

well described by just three Stokeslets [23]. In-plane versions of these models recapitulated the stochastic (run-and-tumble) character of biflagellar coordination [24-27]. These minimal representations of ciliary beating as beads moving along a prescribed orbits are powerful tools for studying hydrodynamic synchronization [28-30].

Unlike in previous studies, we allow the flagellar beads to rotate out of the plane. This introduces a nonzero tilt angle $\beta$ between the flagellar orbital planes $\pi_{( \pm \beta)}$ and the frontal $\boldsymbol{e}_{x}^{\prime}-\boldsymbol{e}_{z}^{\prime}$ plane, which we hypothesize suffices to produce both axial rotation and helical swimming [Fig. 1(b)]. The flagella orbits are centered at $\boldsymbol{s}_{i}=\boldsymbol{r}_{0}+(-1)^{i} \ell \boldsymbol{e}_{x}^{\prime}+h \boldsymbol{e}_{z}^{\prime}, \quad i=1$, 2. Flagellar beads, located at $\boldsymbol{r}_{i}=\boldsymbol{s}_{i}+R \boldsymbol{n}_{i}$, with $\boldsymbol{n}_{i}=(-1)^{i+1} \cos \varphi_{i} \boldsymbol{e}_{x}^{\prime}+$ $(-1)^{i+1} \sin \beta \sin \varphi_{i} \boldsymbol{e}_{y}^{\prime}+\cos \beta \sin \varphi_{i} \boldsymbol{e}_{z}^{\prime}$, rotate with phases $\varphi_{i}$. For breaststrokes, we require $0 \leq \beta<\pi / 2, \dot{\varphi}_{i} \geq 0$. The body axes $\boldsymbol{e}_{i}^{\prime}$ transform to the lab frame $\boldsymbol{e}_{i}$ via Euler angles $\boldsymbol{\theta}=\left(\theta_{1}, \theta_{2}, \theta_{3}\right)$ (pitch, yaw, and roll). The swimmer kinematics are fully described by $\boldsymbol{X}=\left(x_{0}, y_{0}, z_{0}, \theta_{1}\right.$, $\left.\theta_{2}, \theta_{3}, \varphi_{1}, \varphi_{2}\right)$ and the parameters $h, \ell, \beta, R, a_{0}, a$. We impose force- and torque-free conditions: $\boldsymbol{F}_{0}+\boldsymbol{F}_{1}+\boldsymbol{F}_{2}=$ $\mathbf{0}$ and $\boldsymbol{T}_{0}+\boldsymbol{T}_{1}+\boldsymbol{T}_{2}=\mathbf{0}$, where $\boldsymbol{T}_{i}=\boldsymbol{r}_{i} \times \boldsymbol{F}_{i}+\overline{\boldsymbol{T}}_{i}$, and $\overline{\boldsymbol{T}}_{i}$ is the intrinsic torque due to the $i$ th sphere's rotation around an internal axis. Since $a \ll a_{0}$, we assume that $\overline{\boldsymbol{T}}_{1,2} \ll \overline{\boldsymbol{T}}_{0}$. The swimmer moves with velocity $\boldsymbol{V}=\dot{\boldsymbol{r}}_{0}$ and angular velocity $\boldsymbol{\Omega}$. Using the Oseen approximation for hydrodynamic interactions between beads [31], we have

$$
\begin{aligned}
& \boldsymbol{V}=\left[\boldsymbol{G}\left(r_{01}\right)-\gamma_{0}^{-1}\right] \cdot \boldsymbol{F}_{1}+\left[\boldsymbol{G}\left(r_{02}\right)-\gamma_{0}^{-1}\right] \cdot \boldsymbol{F}_{2}, \\
& \dot{\boldsymbol{r}}_{1}=\gamma_{1}^{-1} \boldsymbol{F}_{1}+\left[\boldsymbol{G}\left(r_{12}\right)-\boldsymbol{G}\left(r_{10}\right)\right] \cdot \boldsymbol{F}_{2}-\boldsymbol{G}\left(r_{10}\right) \cdot \boldsymbol{F}_{1}, \\
& \dot{\boldsymbol{r}}_{2}=\gamma_{2}^{-1} \boldsymbol{F}_{2}+\left[\boldsymbol{G}\left(r_{21}\right)-\boldsymbol{G}\left(r_{20}\right)\right] \cdot \boldsymbol{F}_{1}+\boldsymbol{G}\left(r_{20}\right) \cdot \boldsymbol{F}_{2}, \\
& \boldsymbol{\Omega}=\gamma_{0 r}^{-1} \overline{\boldsymbol{T}}_{0}=-\gamma_{0 r}^{-1}\left(\boldsymbol{T}_{1}+\boldsymbol{T}_{2}\right),
\end{aligned}
$$

where $\boldsymbol{r}_{i j}=\boldsymbol{r}_{i}-\boldsymbol{r}_{j}, \quad \gamma_{i}=6 \pi \eta a_{i}, \quad \gamma_{0 r}=8 \pi \eta a_{0}^{3}, \quad \boldsymbol{G}(\boldsymbol{r})=$ $(\mathbb{I}+\hat{\boldsymbol{r}} \otimes \hat{\boldsymbol{r}}) /(8 \pi \eta)$, and the dot denotes a time derivative. These reduce to a set of ten equations for unknowns $\left(\boldsymbol{V}, \boldsymbol{\Omega}, \dot{\varphi}_{1,2}, F_{1,2}^{(n)}\right)$ (see Supplemental Material [31]). The method of quaternions was used to resolve singularities at $\theta_{2}= \pm \pi / 2$ [32].

Experiments.-Our model aims to link the threedimensional nature of flagellar beating to the cell's freeswimming behavior, but is Chlamydomonas flagellar beating truly nonplanar, and if so, to what extent? A possible out-of-plane component was detected based on manual tracings of flagellar waveforms [16]. The same authors argued that helical swimming could result from transient asynchronies between the two flagella. Yet this is incompatible with recent studies showing that phase slip asynchronies occur randomly, not periodically [26,33]. Here we use live-cell imaging and micromanipulation to prove that Chlamydomonas flagella beat with a persistent, nonplanar pattern in vivo. To better visualize the motion, we gently aspirated cells onto pipettes following previous protocols [33,39]. Cells were repositioned with flagella viewed (at 3000 frames/s) directly from above. The eyespot delineates the cis (closest to the eyespot) from the trans flagella [Fig. 2(a)].

The results reveal that the waveform is inherently nonplanar. The Chlamydomonas breaststroke is usually imaged in the $\boldsymbol{e}_{x}^{\prime}-\boldsymbol{e}_{z}^{\prime}$ plane [Fig. 2(c), inset]; here we observed a significant component of motion in the $\boldsymbol{e}_{x}^{\prime}-\boldsymbol{e}_{y}^{\prime}$ plane [Figs. 2(c)-2(e)]. During the power stroke, the flagella extend and pull away from the body while flagellar tips move in opposite directions; the direction of travel is immediately reversed during each subsequent recovery stroke. Over a stereotypical beat, the tips trace closed orbits [Fig. 2(c)]. The rotary motion generates axial torques, balanced by the micropipette. A freely swimming cell, viewed from above, will rotate clockwise (CW) during the power stroke but counterclockwise (CCW) during the recovery stroke. This is analogous to the "two steps forward, one step backward" interpretation for the in-plane breaststroke, which arises due to flagella drag anisotropy. We found that the rotary motion is also periodic (at the flagellar beat frequency) and is stable over thousands of cycles. We estimated the effective orbital tilt angle $\beta$ from the high-speed videos using trigonometry: $\beta=\sin ^{-1}[(\ell / R) \tan [\Delta \alpha / 2])$, where $\Delta \alpha$ is the angle subtended by the flagellum's uppermost tip in the transverse $\boldsymbol{e}_{x}^{\prime}-\boldsymbol{e}_{y}^{\prime}$ plane [31]. For $N=6$ distinct cells, $\sim 10^{3}$ 

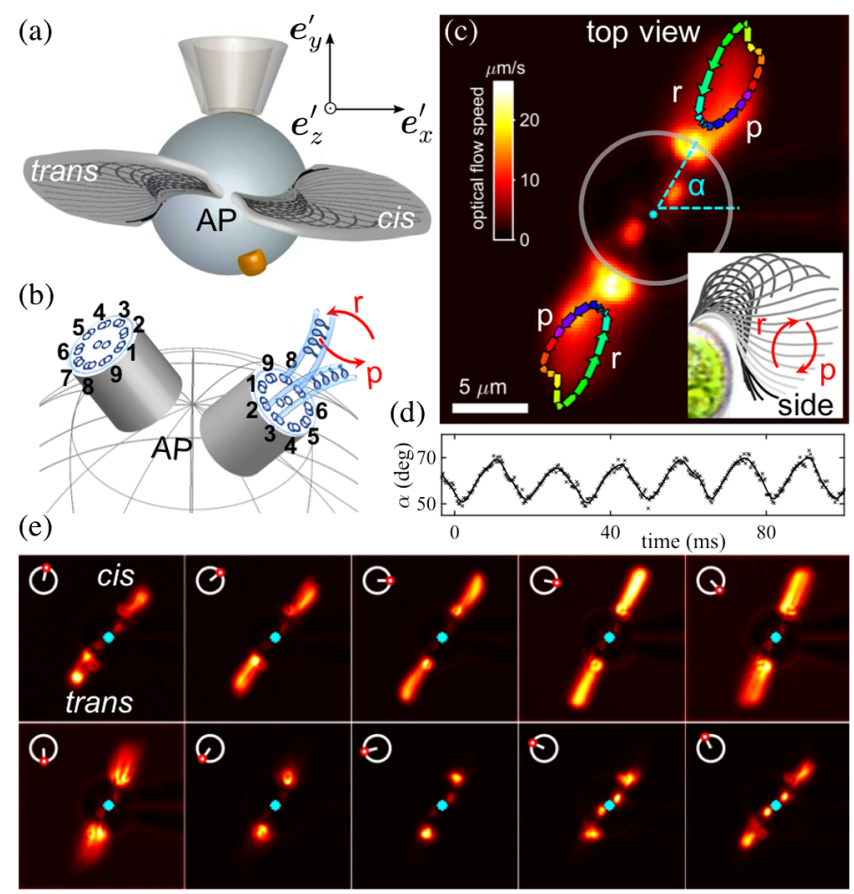

FIG. 2. Nonplanar flagellar beating in Chlamydomonas (Supplemental Material, Video 1 [31]). (a) Cells aspirated onto pipettes are imaged from the anterior pole (AP). An eyespot is located $\sim 45^{\circ}$ from the mean beat plane. (b) The observed screwing motion of the flagella is coincident with the rotational symmetry of the basal apparatus, particularly, the chiral ordering of microtubule doublets $1 \rightarrow 9$. Known axonemal heterogeneities segregate the dyneins into two groups, those on doublets 2-4 operate for the power $(p)$ stroke, but 6-8 for the recovery $(r)$ stroke [40]. (c) Waveforms tracked by optic flow (arrows follow tip rotation) show a small offset [31] between the basal bodies. The instantaneous beat plane (defined by $\alpha$ ) rotates periodically (d). Waveforms are ordered by phase [41] and averaged over $\sim 1000$ consecutive cycles to show the phase dependence of the nonplanar beat pattern (e).

consecutive beat cycles per individual, we estimated $\beta=0.3 \mathrm{rad} \approx 17.2^{\circ}$ (see summary statistics in Table 1 in the Supplemental Material [31]).

Simulations.-We input this estimate of $\beta$ into our threebead model with cell-realistic parameters to determine if beat nonplanarity can indeed generate axial rotation and helical swimming. All lengths are nondimensionalized by $\ell$ (=10 $\mu \mathrm{m}$, typical cell size), forces by the average tangential flagellar force $F_{0}(=30 \mathrm{pN}$, typical force produced by

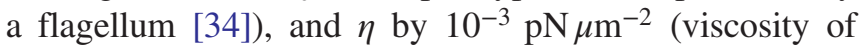
water). When $\beta=0$ (no tilt), pitch and roll $\left(\theta_{1,3}\right)$ are suppressed, and our model reduces to the purely planar case investigated by other authors $[24,42]$. This is when the only nonvanishing component of torque is along $\boldsymbol{e}_{y}^{\prime}$ [Eq. (4)] and swimming is constrained to the cell's frontal plane $\left(\Omega_{y}\right.$, yaw). For a nonplanar beat $(\beta \neq 0)$, there is a nonvanishing resultant axial torque $T_{z}$. Equal driving forces $\left(F_{1}^{(t)}=F_{2}^{(t)}\right)$ produce purely axial rotation, since other components of $\boldsymbol{\Omega}$ cancel by symmetry. The resulting trajectory is linear, with $\boldsymbol{\Omega} \| \boldsymbol{V}$. The trajectory can be a nondegenerate helix only if $\boldsymbol{\Omega} \# \boldsymbol{V}$. Rotational symmetry about $\boldsymbol{e}_{z}^{\prime}$ can be broken if (1) the scaffold shape is asymmetric, (2) flagella planes have different tilt $\left(\beta_{1} \neq \beta_{2}\right)$, and (3) flagella experience different driving forces $\left(F_{1}^{(t)} \neq F_{2}^{(t)}\right)$. The first two cases can produce irregular and nonhelical trajectories. We focus here on case 2 and only consider asymmetric force profiles of the form $F_{i}^{(t)}=1+c_{i} \cos \varphi_{i}[30,43]$.

For $\beta>0$, symmetry breaking introduced by hydrodynamic interactions between the beads results in either forward- or backward-CCW swimming at different phases of the beat cycle. The cycle-averaged swimming speed is given by $\overline{\boldsymbol{V}}=\left(1 / T_{b}\right) \int_{0}^{T_{b}} \boldsymbol{V}(t) d t=\left(1 / T_{b}\right)$ $\int_{0}^{2 \pi}[\boldsymbol{V}(\bar{\varphi}) / \dot{\bar{\varphi}}] / d \bar{\varphi}$ with flagellar period $T_{b}$ and $\bar{\varphi}=\left(\varphi_{1}+\varphi_{2}\right) / 2$, with an analogous expression for angular velocity $\overline{\boldsymbol{\Omega}}$ [31]. The dynamical system described by Eqs. (2) and (3) is highly sensitive to swimmer shape. The strength of the hydrodynamic interactions between flagella beads are dictated by scaffold parameters, which determine net swimming (and rotation) direction per cycle, as in the 2D case $[25,43]$. We choose configurations where $\bar{V}_{z}>0, \bar{\Omega}_{z}<0$ (CCW is positive), and average flagella beat frequencies $f_{b}$ and axial rotation $\bar{\Omega}_{z}$ fall within experimental values. See Supplemental Material [31] for further exploration of parameter space.

We simulate free-swimming trajectories (Fig. 3) for a fixed scaffold shape $\left(a_{0}=0.53, h=1.30, R=0.60\right.$, $a=0.05$ ), tilt angle $\beta=0.3$, but different values of force asymmetry $\Delta F=c_{2}-c_{1}$. Figure 3(a) shows purely axial rotation $(\Delta F=0)$. When $\Delta F \neq 0$, trajectories are superhelical. Superhelices emerge as general solutions of all low-Re swimming dynamics driven by periodic deformations [11] and have been observed in sperm swimming and in biaxial self-propelled particles under external torques [44]. A $1 \%$ difference in $\Delta F$ suffices to generate realistic superhelical trajectories [Fig. 3(b)]. Here, the average trajectory of the centroid $\boldsymbol{r}_{0}$ prescribes an outer helix with a pitch of $\sim 95$ and radius $\sim 5.9 \mu \mathrm{m}$, while "fast" helical swirls appear on the timescale of the flagellar period $T_{b}=f_{b}^{-1} \approx 17 \mathrm{~ms}$. Less regular helices emerge with higher asymmetry [Figs. 3(c) and 3(d)]. Frequencies of mean flagellar bead rotation $f_{b}$ and axial rotation $\Omega_{z}$ vary with increasing orbital tilt angle $\beta \in(0,2 \pi)$ [Fig. 3(e)]. As expected, axial rotation velocity increases with beat nonplanarity (higher $\beta$ ). A tilt angle $\beta \approx 0.3$ corresponds to a rotation frequency of $1-2 \mathrm{~Hz}$. We conclude that realistic helical swimming with a symmetric scaffold shape can be achieved using a very small force asymmetry between flagella, together with a small orbital tilt.

Phototactic steering.-Can cells exploit asymmetric flagellar driving for trajectory control? We hypothesize that this mechanism underlies phototaxis. Chlamydomonas 

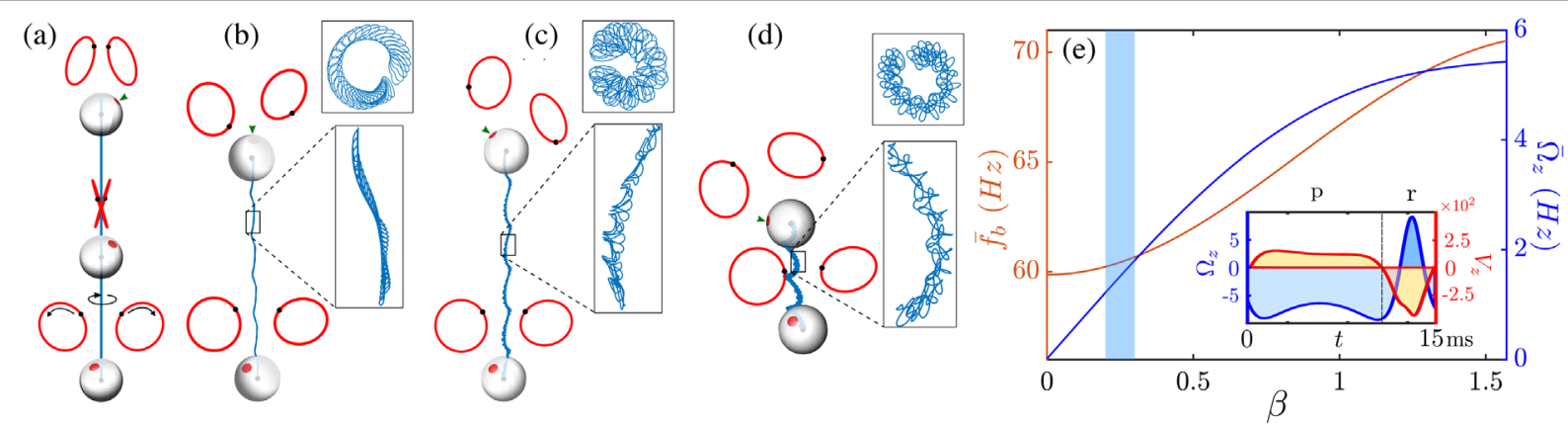

FIG. 3. Nonplanar flagellar beating leads to superhelical trajectories (eyespot, small arrowhead). For the same geometry $a_{0}=0.53$, $h=1.30, R=0.60, \beta=0.30, a=0.05$, examples show (a) purely axial rotation when $c_{1,2}=0.7$, (b) "smooth" superhelix for $c_{1}=0.7, c_{2}=0.71$, (c) a superhelix with irregular inner helix for asymmetrical driving forces $c_{1}=0.6, c_{2}=0.62$, and (d) a more irregular trajectory for $c_{1}=0.3, c_{2}=0.5$. (e) Mean beat frequency of the two flagella (orange) and axial component of angular velocity (blue) as functions of $\beta$ (numerical data fitted with sum of sines). The shaded region marks range of $\beta$ measured in experiments (see Supplemental Material [31]). Inset: linear and angular velocities (in $\mu \mathrm{m} / \mathrm{s}$ and hertz) over one beat, note the CW-forward motion during the power $(p)$ stoke and CCW-backward motion during the recovery $(r)$ stroke.

can perform positive or negative phototaxis, depending on the stimulus $[19,45]$. Phototactic steering is associated with changes in both beat amplitude and frequency and is fine-tuned to the body rotation frequency $[16,20,46]$. Steering is accomplished by changing $\boldsymbol{\Omega}[10,47]$, which in turn changes helix properties (radius, pitch, orientation). Here, $\boldsymbol{\Omega}$ and $f_{b}$ are strongly coupled. To simulate phototaxis (Fig. 4), we assume that a light stimulus I either attenuates or accentuates $\Delta F$ depending on the alignment between the eyespot and stimulus [48]. Denoting by $\hat{\mathbf{N}}$ the vector normal to the eyespot surface, the intensity incident on the eyespot is $I(t)=I_{0}(t) \cos \phi(t) H[\cos \phi(t)]$, where $\cos \phi=\hat{\mathbf{N}} \cdot \hat{\mathbf{I}}=$ $\left(\boldsymbol{e}_{x}^{\prime}-\boldsymbol{e}_{y}^{\prime}\right) \cdot\left(-\boldsymbol{e}_{y}\right) / \sqrt{2}$, and $H$ is the Heaviside step function. This framework can be applied to any type of taxis in response to a vectorial cue.

Flagella identity is important. When $\Delta F=0.01$ (no signal), the stronger (dominant) flagellum is the cis flagellum $\left(c_{\text {cis }}^{(0)}=0.71, c_{\text {trans }}^{(0)}=0.7\right)$. Force profiles are then modified to $c_{\text {cis,trans }}=c_{\text {cis,trans }}^{(0)} \mp p \log [1+I(t)]$, where $p$ is the sensitivity of biflagellar dominance to the signal. Signs were chosen so that the model flagella responded differentially to the same signal [18] and in a direction compatible with experiments [49]. Importantly, here $\boldsymbol{\Omega}$ emerges from the biflagellar force profiles, and was not assumed a priori to produce a regular helix. The resulting dynamics are characterized by a change in the pitch, radius, and axis of the (super)helical trajectory. The sign of flagellar dominance determines the sign of phototaxis. Compared to the no-light scenario, the cell turns toward the light when $\Delta F$ flips sign (trans dominant), but away when the cis-flagellum becomes more dominant $(\Delta F$ more positive). This indicates that alignment to a vectorial stimulus is indeed possible simply by varying the two relative forcing profiles (Fig. 4).
Concluding remarks.-We showed for the first time that Chlamydomonas flagella beat with a persistent, 3D beat pattern in vivo and quantified this as an orbital tilt. Accounting for this nonplanarity, we developed a threebead hydrodynamic model with cell-realistic geometries,
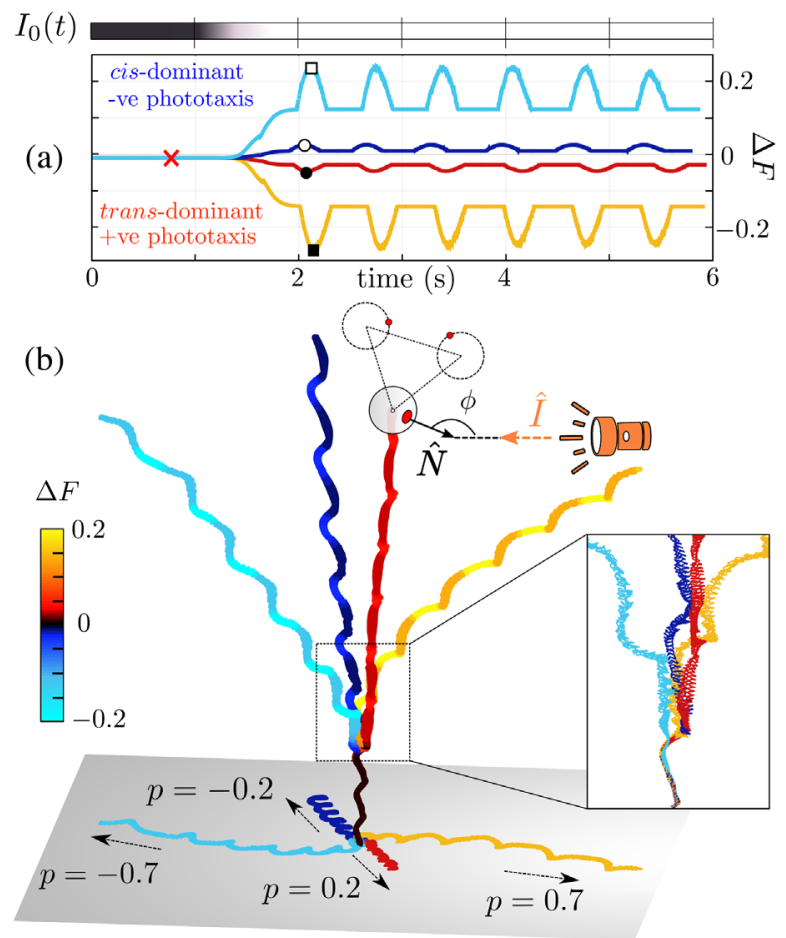

FIG. 4. Flagellar dominance controls phototactic steering. (a) Time series of stimulus intensity $I_{0}$ (gray scale $=$ intensity) and the response $\Delta F$ for four different $p$ values. Markers indicate characteristic trans-cis frequencies: times, 61-63; square, 58-75; filled square, 78-51; circle, 51-73; filled circle, 53-69 Hz. (b) The corresponding $3 \mathrm{D}$ tracks show either positive or negative phototaxis. Color indicates force asymmetry $\Delta F$. In all cases, $a_{0}=0.53, \quad h=1.3, \quad \ell=1, \quad R=0.6, \quad \beta=0.3, \quad a=0.05$, $c_{\text {cis }}(t=0)=0.71, c_{\text {trans }}(0)=0.7$. 
which captures the sense and magnitude of axial rotation of real cells. Superhelical trajectories emerged directly from the flagellar motion and were not prescribed. The model swims more slowly $(15-30 \mu \mathrm{m} / \mathrm{s})$ than live cells $(50-100 \mu \mathrm{m} / \mathrm{s}$ [50]), suggesting limitations of a Stokeslettype swimmer. To account for amplitude-frequency coupling pertaining to real flagella, future work will include elasticity, slender-filament dynamics, and basal coupling [39]. This can help constrain biflagellar synchrony to enhance swimming and steering efficiency.

In Chlamydomonas, the orbital tilt is likely to be fixed genetically. The beat pattern may result from an intrinsically chiral axoneme, which contains heterogeneities that could enforce bend anisotropy [40]. Such an axoneme may not be capable of sustaining perfectly planar beat patterns [11,51]. This view is supported by a recent study [52], which revealed that isolated Chlamydomonas axonemes, when reactivated with adenosine triphosphate (ATP), also beat with a three-dimensional pattern. Moreover, nearly all axonemes displayed the same torsional signature, consistent with the rotational symmetry of the basal apparatus and sense of flagellar rotation we observed [Figs. 2(b) and 2(c)].

A small asymmetry $(1 \%)$ in biflagellar driving forces was necessary and sufficient for helical (not just purely axial) swimming. Whether this is augmented by further structural or shape asymmetries (if $\beta_{1} \neq \beta_{2}$ ) can be revealed by multifocal imaging, to measure the full torsional profile of each flagellum. A small cis-trans waveform asymmetry was already identified in the beat plane [33]. A stimulusdependent asymmetry allowed our simple threebead swimmer to perform phototaxis. Controllable steering is thus fairly insensitive to gait and the precise beating waveform. Indeed some algal biflagellates that do not use in-phase breaststrokes are nonetheless phototactic (e.g., Polytoma, Cryptomonas). Our Letter emphasizes the functional distinction between the two Chlamydomonas flagella, showing that a tunable and reversible biflagellar dominance likely operates in live cells for helical klinotaxis. Consistent with this view, mutant strains (mia, bop5-3, ptxl, and $l s p 1$ ) have "smoother" trajectories and defective or weakened phototaxis [53-55]. Either flagellum can be dominant, depending on the stimulus. Indeed changes in $\mathrm{Ca}^{2+}$ produced opposite responses in reactivated cis and trans axonemes [18,49]. Our results offer key insights into the physiological basis of dynamic sensorimotor control, as implemented in a simple, aneural organism with few morphological asymmetries. An important next step will be to determine how biflagellar dominance is regulated at the molecular level (e.g., of dynein activity).

We thank Ray Goldstein for support during the initial phase of this project and Peter Ashwin and Gáspár Jékely for useful discussions. This work was funded by the European Research Council (ERC) under the European
Union's Horizon 2020 Research and Innovation Programme (Grant No. 853560, EvoMotion) and a Springboard Award from the Academy of Medical Sciences and Global Challenges Research Fund (SBF003\1160) to K. Y. W.

* Corresponding author.

k.y.wan2@exeter.ac.uk

[1] G. Jékely, J. Colombelli, H. Hausen, K. Guy, E. Stelzer, F. Nédélec, and D. Arendt, Mechanism of phototaxis in marine zooplankton, Nature (London) 456, 395 (2008).

[2] T. L. Jahn and J. J. Votta, Locomotion of protozoa, Annu. Rev. Fluid Mech. 4, 93 (1972).

[3] A. D. Drake, Observations on bull sperm rotation, Biol. Reprod. 10, 78 (1974).

[4] E. Lauga, Bacterial hydrodynamics, Annu. Rev. Fluid Mech. 48, 105 (2016).

[5] B. M. Friedrich and F. Jülicher, Steering Chiral Swimmers along Noisy Helical Paths, Phys. Rev. Lett. 103, 068102 (2009).

[6] K. Y. Wan and G. Jékely, Origins of eukaryotic excitability, Philos. Trans. R. Soc. B 376, 20190758 (2021).

[7] H. S. Jennings, On the significance of the spiral swimming of organisms, Am. Nat. 35, 369 (1901).

[8] H. Gadêlha, P. Hernández-Herrera, F. Montoya, A. Darszon, and G. Corkidi, Human sperm uses asymmetric and anisotropic flagellar controls to regulate swimming symmetry and cell steering, Sci. Adv. 6, eaba5168 (2020).

[9] C. Krüger, G. Klös, C. Bahr, and C. C. Maass, Curling Liquid Crystal Microswimmers: A Cascade of Spontaneous Symmetry Breaking, Phys. Rev. Lett. 117, 048003 (2016).

[10] F. Lancia, T. Yamamoto, A. Ryabchun, T. Yamaguchi, M. Sano, and N. Katsonis, Reorientation behavior in the helical motility of light-responsive spiral droplets, Nat. Commun. 10, 5238 (2019).

[11] M. Rossi, G. Cicconofri, A. Beran, G. Noselli, and A. De Simone, Kinematics of flagellar swimming in Euglena gracilis: Helical trajectories and flagellar shapes, Proc. Natl. Acad. Sci. U.S.A. 114, 13085 (2017).

[12] A. C. H. Tsang, A. T. Lam, and I. H. Riedel-Kruse, Polygonal motion and adaptable phototaxis via flagellar beat switching in the microswimmer Euglena gracilis, Nat. Phys. 14, 1216 (2018).

[13] H. De Maleprade, F. Moisy, T. Ishikawa, and R.E. Goldstein, Motility and phototaxis of gonium, the simplest differentiated colonial alga, Phys. Rev. E 101, 022416 (2020).

[14] A. Gong, S. Rode, U. B. Kauppl, G. Gompper, J. Elgeti, B. M. Friedrich, and L. Alvarez, The steering gaits of sperm, Phil. Trans. R. Soc. B 375, 20190149 (2020)

[15] H. Ito, T. Omori, and T. Ishikawa, Swimming mediated by ciliary beating: Comparison with a squirmer model, J. Fluid Mech. 874, 774 (2019).

[16] U. Rüffer and W. Nultsch, High-speed cinematographic analysis of the movement of Chlamydomonas, Cell Motil. 5, 251 (1985). 
[17] K. Schaller, R. David, and R. Uhl, How Chlamydomonas keeps track of the light once it has reached the right phototactic orientation, Biophys. J. 73, 1562 (1997).

[18] R. Kamiya and G. B. Witman, Submicromolar levels of calcium control the balance of beating between the two flagella in demembranated models of Chlamydomonas, J. Cell Biol. 98, 97 (1984).

[19] K. W. Foster and R. D. Smyth, Light antennas in phototactic algae, Microbiol. Rev. 44, 572 (1980).

[20] K. Josef, J. Saranak, and K. W. Foster, Ciliary behavior of a negatively phototactic Chlamydomonas reinhardtii, Cell Motil. Cytoskeleton 61, 97 (2005).

[21] R. J. Wheeler, Use of chiral cell shape to ensure highly directional swimming in Trypanosomes, PLoS Comput. Biol. 13, e1005353 (2017).

[22] T. Fenchel, How dinoflagellates swim, Protist 152, 329 (2001).

[23] K. Drescher, R. E. Goldstein, N. Michel, M. Polin, and I. Tuval, Direct Measurement of the Flow Field around Swimming Microorganisms, Phys. Rev. Lett. 105, 168101 (2010).

[24] R. R. Bennett and R. Golestanian, Emergent Run-andTumble Behavior in a Simple Model of Chlamydomonas with Intrinsic Noise, Phys. Rev. Lett. 110, 148102 (2013).

[25] K. Polotzek and B. M. Friedrich, A three-sphere swimmer for flagellar synchronization, New J. Phys. 15, 045005 (2013).

[26] R. E. Goldstein, M. Polin, and I. Tuval, Noise and Synchronization in Pairs of Beating Eukaryotic Flagella, Phys. Rev. Lett. 103, 168103 (2009).

[27] E. Lushi, V. Kantsler, and R. E. Goldstein, Scattering of biflagellate microswimmers from surfaces, Phys. Rev. E 96, 023102 (2017).

[28] A. Vilfan and F. Jülicher, Hydrodynamic Flow Patterns and Synchronization of Beating Cilia, Phys. Rev. Lett. 96, 058102 (2006).

[29] N. Bruot and P. Cicuta, Realizing the physics of motile Cilia synchronization with driven colloids, Annu. Rev. Condens. Matter Phys. 7, 323 (2016).

[30] D. R. Brumley, M. Polin, T. J. Pedley, and R. E. Goldstein, Hydrodynamic Synchronization and Metachronal Waves on the Surface of the Colonial Alga Volvox Carteri, Phys. Rev. Lett. 109, 268102 (2012).

[31] See Supplemental Material at http://link.aps.org/ supplemental/10.1103/PhysRevLett.126.088003 for further details and results, which includes Refs. [24,25,32-38].

[32] M. Boyle, The integration of angular velocity, Adv. Appl. Clifford Algebras 27, 2345 (2017).

[33] K. Y. Wan, K. C. Leptos, and R. E. Goldstein, Lag, lock, sync, slip: The many "phases" of coupled flagella, J. R. Soc. Interface 11, 20131160 (2014).

[34] T. J. Böddeker, S. Karpitschka, C. T. Kreis, Q. Magdelaine, and O. Bäumchen, Dynamic force measurements on swimming Chlamydomonas cells using micropipette force sensors, J. R. Soc. Interface 17, 20190580 (2020).

[35] J. Schindelin, I. Arganda-Carreras, E. Frise, V. Kaynig, M. Longair, T. Pietzsch, S. Preibisch, C. Rueden, S. Saalfeld, B. Schmid et al., Fiji: An open-source platform for biological-image analysis, Nat. Methods 9, 676 (2012).
[36] J. Diebel, Representing attitude: Euler angles, unit quaternions, and rotation vectors, Matrix 58, 1 (2006).

[37] M. Reichert, Doctoral thesis, University of Constance, 2006.

[38] N. Yubuki and B.S. Leander, Evolution of microtubule organizing centers across the tree of eukaryotes, Plant J. 75, 230 (2013).

[39] K. Y. Wan and R. E. Goldstein, Coordinated beating of algal flagella is mediated by basal coupling, Proc. Natl. Acad. Sci. U.S.A. 113, E2784 (2016).

[40] H. J. Hoops and G. B. Witman, Outer doublet heterogeneity reveals structural polarity related to beat direction in Chlamydomonas flagella, J. Cell Biol. 97, 902 (1983).

[41] K. Y. Wan and R. E. Goldstein, Rhythmicity, Recurrence, and Recovery of Flagellar Beating, Phys. Rev. Lett. 113, 238103 (2014).

[42] B. M. Friedrich and F. Jülicher, Flagellar Synchronization Independent of Hydrodynamic Interactions, Phys. Rev. Lett. 109, 138102 (2012).

[43] R. R. Bennett and R. Golestanian, Phase-dependent forcing and synchronization in the three-sphere model of Chlamydomonas, New J. Phys. 15, 075028 (2013).

[44] R. Wittkowski and H. Löwen, Self-propelled Brownian spinning top: Dynamics of a biaxial swimmer at low Reynolds numbers, Phys. Rev. E 85, 021406 (2012).

[45] K. Wakabayashi, Y. Misawa, S. Mochiji, and R. Kamiya, Reduction-oxidation poise regulates the sign of phototaxis in Chlamydomonas reinhardtii, Proc. Natl. Acad. Sci. U.S.A. 108, 11280 (2011).

[46] K. C. Leptos, M. Chioccioli, S. Furlan, A. I. Pesci, and R. E. Goldstein, An adaptive flagellar photoresponse determines the dynamics of accurate phototactic steering in Chlamydomonas, bioRxiv: 254714 (2018).

[47] H. C. Crenshaw, Orientation by helical motion I. Kinematics of the helical motion of organisms with up to six degrees of freedom, Bull. Math. Biol. 55, 197 (1993).

[48] R. R. Bennett and R. Golestanian, A steering mechanism for phototaxis in Chlamydomonas, J. R. Soc. Interface 12, 20141164 (2015).

[49] N. Isogai, R. Kamiya, and K. Yoshimura, Dominance between the two flagella during phototactic turning in Chlamydomonas, Zoological science 17, 1261 (2000).

[50] V. A. Martinez, R. Besseling, O. A. Croze, J. Tailleur, M. Reufer, J. Schwarz-Linek, L. G. Wilson, M. A. Bees, and W. C. Poon, Differential dynamic microscopy: A highthroughput method for characterizing the motility of microorganisms, Biophys. J. 103, 1637 (2012).

[51] P. Sartori, V.F. Geyer, A. Scholich, F. Jülicher, and J. Howard, Dynamic curvature regulation accounts for the symmetric and asymmetric beats of Chlamydomonas flagella, eLife 5 (2016), .

[52] S. Mojiri, S. Isbaner, S. Mühle, H. Jang, A. J. Bae, I. Gregor, A. Gholami, and J. Enderlein, Three-dimensional beating dynamics of chlamydomonas flagella, BioRxiv, (2020), https://doi.org/10.1101/2020.07.20.212159.

[53] K. E. VanderWaal, R. Yamamoto, K. Wakabayashi, L. Fox, R. Kamiya, S. K. Dutcher, P. V. Bayly, W. S. Sale, and M. E. Porter, bop5 mutations reveal new roles for the IC138 phosphoprotein in the regulation of flagellar motility and asymmetric waveforms, Mol. Biol. Cell 22, 2862 (2011). 
[54] K. C. Leptos, K. Y. Wan, M. Polin, I. Tuval, A. I. Pesci, and R. E. Goldstein, Antiphase Synchronization in a FlagellarDominance Mutant of Chlamydomonas, Phys. Rev. Lett. 111, 158101 (2013).
[55] N. Okita, N. Isogai, M. Hirono, R. Kamiya, and K. Yoshimura, Phototactic activity in Chlamydomonas "non-phototactic" mutants deficient in $\mathrm{Ca}^{2+}$-dependent control of flagellar dominance or in inner-arm dynein, J. Cell Sci. 118, 529 (2005). 